\title{
Role of Manual Immature to Total Neutrophil (I/T) Ratio and Automated Immature Granulocyte Count (IGC) and Percentage (IG\%) in the Early Diagnosis of Neonatal Sepsis
}

\section{Nalika Jayasekara}

University of Sri Jayewardenepura,Sri Lanka

Chandima Kulathilake ( $\nabla$ kulathilake@sjp.ac.lk)

University of Sri Jayewardenepura,Sri Lanka

\section{Saraji Wijesekara}

University of Sri Jayewardenepura,Sri Lanka

Indira Wijesiriwardena

University of Sri Jayewardenepura,Sri Lanka

\section{Research Article}

Keywords: neonatal sepsis, manual I/T ratio, automated immature granulocytes

Posted Date: January 29th, 2021

DOl: https://doi.org/10.21203/rs.3.rs-154297/v1

License: (c) (1) This work is licensed under a Creative Commons Attribution 4.0 International License.

Read Full License 
Role of Manual Immature to Total neutrophil (I/T) ratio and Automated Immature Granulocyte count (IGC) and percentage (IG\%) in the early diagnosis of neonatal sepsis

\section{Running title: Role of Immature Granulocyts in the early diagnosis of neonatal sepsis}

Nalika Jayasekara $^{1}$, Chandima Kulathilake ${ }^{1}$, Saraji Wijesekara ${ }^{2}$, Indira Wijesiriwardena ${ }^{1}$

${ }^{1}$ Department of Pathology, Faculty of Medical Sciences, University of Sri

Jayewardenepura, Sri Lanka

${ }^{2}$ Department of Paediatrics, Faculty of Medical Sciences, University of Sri

Jayewardenepura, Sri Lanka

\section{Corresponding Author}

Dr. Chandima Kulathilake

Consultant Haematologist/Senior Lecturer

Department of Pathology, Faculty of Medical Sciences

University of Sri Jayewardenepura

Sri Lanka

Email: kulathilake@sjp.ac.lk

Contact: +94 714966803 
Word count

Abstract 245

Text 2361 (excluding title page, abstract, tables and references)

References - 13 


\title{
KEY WORDS
}

neonatal sepsis, manual I/T ratio, automated immature granulocytes

\begin{abstract}
Background: The diagnosis of neonatal sepsis is challenging due to non-specific and subtle clinical features, low sensitivity and delay in routine laboratory tests. Current study was conducted to evaluate the role of manual immature/total (I/T) neutrophil ratio and automated immature granulocyte count (IGC) and immature granulocyte percentage $(\mathrm{IG} \%)$ in the diagnosis of neonatal sepsis.
\end{abstract}

Materials and Methods: An analytical cross-sectional study was done during a period of 6 months with a sample of 55 neonates admitted to Colombo South Teaching Hospital, Sri Lanka. A combination of clinical and laboratory parameters including full blood count, C-reactive protein and blood culture were used to identify the neonates with probable sepsis. The population was subcategorized into five (5) groups and manual immature/total neutrophil (I/T) ratio, immature granulocyte count (IGC) and immature granulocyte\%(IG\%) were done in each neonate.

Results: The sensitivity of manual I/T ratio was $93.75 \%$ and negative predictive value (NPV) was $95.24 \%$. The sensitivity for lower cut off values, IGC of $0.03 \times 10^{3} / \mu \mathrm{L}$ and IG\% of $0.5 \%$ was $80 \%$ and $73.33 \%$ respectively. The NPV for above cut-off values were $25 \%$ and $0.5 \%$ respectively. The NPV was improved with higher cut-off values with $70.90 \%$ for IGC 0.3 and $70.59 \%$ for IG $3 \%$, but sensitivity remained low with $40 \%$ and $33.33 \%$ respectively.

Conclusion: Manual I/T ratio remains as a useful diagnostic tool in diagnosing and excluding neonatal sepsis with a very good sensitivity and NPV. However, further studies and well defined reference intervals are required in automated IGC and IG\%. 


\section{INTRODUCTION}

Early onset sepsis is an important cause of morbidity and mortality in neonates. ${ }^{1,2,3}$ The diagnosis is challenging due to non-specific and subtle clinical features in neonates. ${ }^{4}$ Although the incidence is low (1.4-3.2\% increasing with decreasing gestational age) the severity of the disease and non-specific nature of early symptoms leads to evaluate and treat many more neonates who actually did not have the disease. ${ }^{5}$ In routine practice the diagnosis of neonatal sepsis is by high clinical suspicion supported by laboratory parameters which include positive blood culture, elevated C-Reactive Protein(CRP) and high or low WBC count with immature granulocytes in the peripheral blood smear.

As there are so many drawbacks with persisting laboratory investigations, a test which gives quick results with a good sensitivity, high negative predictive value and cost effective is a need. Immature to total neutrophil ratio (I/T ratio) which is defined as the proportion of the number of immature cells including blasts, promyelocytes, myelocytes and band forms to the number of total netrophils determined in a 100 cell-manual differential count in a peripheral blood smear has a long clinical tradition in the diagnosis of early neonatal sepsis. ${ }^{1-10}$ Several studies have proved that an elevated IT ratio of $>0.2$ has a very high negative predictive value (some close to 100\%) and a good sensitivity rate. ${ }^{1-10}$

Automated immature granulocyte count (IGC) and immature granulocyte percentage (IG\%), are available in modern haematology analyzers and considered as precise and accurate as drawbacks such as inter- and intra observer variation of microscopic band cell identification in the above manual differential count is obviated.

In Sri Lanka previous studies on this topic were not available, so that our study wanted to find out the utility of these parameters in a cohort of neonates in our country. We evaluated the significance of immature/total neutrophil (I/T) ratio and automated immature granulocyte count (IGC) and percentage (IG\%) in the early diagnosis of neonatal sepsis. 


\section{MATERIALS AND METHODS}

This was an analytical cross-sectional study conducted over a period of six months. The study group included babies < 14 days old who were admitted to special care baby unit(SCBU) and post natal ward of professorial unit of Colombo South Teaching Hospital during the study period. Neonates who were suspected to have sepsis based on peri-natal risk factors, prematurity and clinical features were taken as the study group.

A combination of clinical and lab parameters was used to identify the neonates with presumed sepsis. The clinical evaluation based on peri-natal risk factors and clinical features (eg: lethargy, poor feeding, change in temperature and respiratory distress) for probable sepsis was done by a consultant pediatrician.

In neonates with clinically presumed sepsis, samples were taken for 0 hour blood culture before starting antibiotics under sterile conditions into BD BACTEC PEDS PLUS blood culture bottles. Samples were incubated in the BD BACTEC fluorescent series instrument followed by manual plating for positive BACTEC samples.

The samples for full blood count (FBC) were taken at $24 \mathrm{hrs}$ into microtainer tripotassium EDTA tubes (approximately $0.5 \mathrm{ml}$ of blood by venepuncture) and analyzed in the Mindray BC 6800 analyzer in the manual mode within 04 hours of collection to obtain total WBC count, immature granulocyte count(IGC) and immature granulocyte\%(IG\%). Mindray BC 6800 IGC included promyelocytes, myelocytes and metamyelocytes(not band forms). Those immature forms were detected by the machine using three principles; (1) Forward scatter for cell volume, (2) side scatter for granularity and (3) fluorescence with high affinity for nucleic acid, and when excited by a $635 \mathrm{~nm}$ laser beam, the cells fluorescence proportional to their content of nucleic acid. IGs show an intense fluorescence from which they can be separated from mature neutrophils. The machine was calibrated and a quality control sample for WBC and IGs was analyzed daily.

Blood smears were made manually, stained with leishman stain and two 100-cell manual differential counts were done using oil immersion object of Olympus $\mathrm{CH}-30$ microscope 
at a final magnification of 1000. The manual IGC included promyelocytes, myelocytes, metamyelocytes and band forms. Immature/Total neutrophil (I/T) ratio was calculated for each smear.

The samples for CRP were taken at $48 \mathrm{hrs}$ into plain bottles and sent for analysis.

As there was no single laboratory parameter available as the diagnostic tool in early neonatal sepsis; high clinical suspicion, positive blood culture and increased CRP value (at 48 hours) were considered. Having high clinical suspicion and at least one of the above laboratory parameters were considered as proven sepsis.

The neonates were further categorized into 05 groups after the results of blood culture and CRP as (1)neonates with definitive sepsis with positive blood culture, (2)neonates with probable sepsis with strong clinical history and/or positive CRP but negative blood culture, (3)neonates who were investigated and treated but no proven sepsis, (4)neonates who were investigated but not treated for sepsis and (5) sepsis not suspected and FBC taken for other reasons. We did not include CSF culture as a laboratory parameter in our study as in early neonatal sepsis work up, CSF analysis is done as a second line investigation, only when there is a clinical suspicion of CNS sepsis (eg: fits or other neurological signs) or if there is positive blood culture. Urine culture was also not included as in newborn babies, uro-sepsis is not common and it is done as a part of septic screen in neonates $>14$ days old.

As per manual method, $\geq 0.2$ was considered as positive I/T ratio. The reference intervals for paediatric population in automated IGC and IG\% were not well defined. Therefore, adult reference ranges were considered, though the literature survey had highlighted two different cut off values for IGC (>0.03 and 0.3$)$ and IG\% (>0.5\% and >3\%).

Sensitivity and Negative Predictive Value (NPV) were considered to be calculated as the two key performance Characteristics. 
Sensitivity (also called the true positive rate or probability of detection) measures the proportion of actual positives that are correctly identified as such. It was calculated using following formula,

Sensitivity $\%=$ True positive $(\mathrm{TP}) /$ True positive $(\mathrm{TP})+$ False negative $(\mathrm{FN}) \times 100 \%$

Negative predictive value is defined as the proportion of true negatives out of negative results. The following formula was used to calculate Negative predictive value,

NPV\% $=$ True negative $(\mathrm{TN}) /$ True negative $(\mathrm{TN})+$ False negative $(\mathrm{FN}) \times 100 \%$

\section{RESULTS}

The study concentrated on blood culture samples that were taken on day 0 before starting antibiotics, FBC taken within 24 hours and the CRP taken at 48 hours. Within the study period, 51 babies were included, and there were 14 neonates with $<34$ weeks of age (extreme premature), 09 were between 34-37 weeks and the rest were term babies.

In the study group of 51 neonates, 45 babies had partial septic screen and six (6) had FBC for other reasons eg: jaundice due to haemolysis. However out of 45 who had partial septic screen, only 39 neonates were started on antibiotics for suspected sepsis. Finally, 19 were diagnosed with proven sepsis including 18 culture negative proven sepsis and one culture positive proven sepsis. It was highlighted that there was only one positive blood culture reported in this population group, which was $1.96 \%$ of total population and $5.26 \%$ of proven sepsis (1 out of 19$)$. (Table 1 )

Out of the 45 neonates who were investigated for suspected sepsis, 20 (39.22\%) were treated with antibiotics initially, became negative in septic screen later and were discontinued on antibiotics.

A total of 42 samples were analyzed for manual I/T ratio $(11+31)$ and 40 samples by automated IGC and IG\% (31+09) according to the data given in Table 2. 
Out of the 42 samples analyzed for manual I/T ratio, 21 had I/T ratio of $>0.2$ (positive). Among them 15 were finally diagnosed with sepsis (TP) and 6 were negative (FP)(Table 3).The sensitivity and NPV for the manual I/T ratio were $93.75 \%$ and $95.24 \%$ respectively.Five out of six of those false positive babies were extremely premature $(<34$ weeks of POA) and one term baby but with congenital kidney disease.

Forty (40) out of 51 samples were analyzed for automated IGC with the cut-off value of 0.03 (Table 4) and 0.3, (Table 5) giving different results. The sensitivity for the two cut off values were $80.00 \%$ and $40.00 \%$ respectively. In contrast, the NPV rose from $25.00 \%$ to $70.90 \%$ with IGC of 0.3 (Table 6).

Similarly, for IG\% in automated method, the sensitivity at cut off of $0.5 \%$ was $73.33 \%$ and the NPV was $42.86 \%$. When the cut-off was raised upto $3 \%$, the sensitivity was $33.33 \%$ and NPV was $70.59 \%$ (Tables $7,8,9$ ).

\section{DISCUSSION}

As mentioned earlier, diagnosis of neonatal sepsis is challenging due to the non-specific and subtle nature of the clinical picture. In routine practice, there is no single laboratory parameter that is used to diagnose neonatal sepsis. Therefore, high clinical suspicion with other laboratory parameters namely, positive blood culture, blood count parameters and positive CRP at 48 hours are used. ${ }^{11,12}$ Out of the laboratory parameters, blood culture positivity is considered as the gold standard. However, this includes several drawbacks including low sensitivity which was also highlighted in the current study, due to inadequate sample volume from the neonates and improper sampling technique and long duration taken for the result (24-48 hours) hence, delaying a prompt diagnosis of sepsis. ${ }^{1-}$ 5 Although CRP is a rapid test, it will take about 24 hours for the neonatal body to produce a significant level of CRP. ${ }^{13}$ 
All these factors lead to unnecessary exposure of healthy neonates who actually do not have sepsis, to antibiotics for minimum of three days. In the current study, the percentage of neonates who were initially started on antibiotics and then discontinued later as no proven sepsis was $39.22 \%$ ( $n=20 / 51)$.

Immature to total neutrophil (I/T) ratio by manual method had been recognized as a useful tool. However, it was not widely used as it was labour intensive. Several studies have proved that an elevated IT ratio of $>0.2$ has a very high negative predictive value (some close to $100 \%$ ) and a good sensitivity rate. ${ }^{1-6}$ The results in our study were reassuring with sensitivity of $93.75 \%$ and NPV of $95.24 \%$ touching the figures in larger cohort studies which indicated 98-100\% (Fig 1).

Considering above factors it is clearly seen that manual I/T ratio is a good screening test to exclude early onset neonatal sepsis. It is still useful in premature babies as there were no false negatives found among premature babies in our study.

Innovations in technology leads automated analyzers in counting immature granulocytes based on light scattering and fluorescence techniques. This gives a solution for main drawbacks in manual I/T ratio such as labour cost, inter- and intra- observer variance in morphology.

However, the reference interval for pediatric age group in automated IGC and IG\% was not well defined. Also in adult reference ranges, two different cut-offs for each were proposed.

In current study, sensitivity for cut off values, IGC 0.03 and IG\% $0.5 \%$ were satisfactory with $80.00 \%$ and $73.33 \%$ respectively. Similarly in the study at Chettinad Deemed University, India, ${ }^{9}$ where the study population included adults, children, infants and neonates, IGC of $0.03 \times 10^{3} / \mu \mathrm{L}$ and IG\% of $0.5 \%$ offered a sensitivity of $86.3 \%$ and $92.2 \%$ respectively $(82 \%$ and $89 \%$ in adults; $88 \%$ and $96 \%$ in children). Measurements were 
Fig 1 : Comparison of Sensitivity and NPV of manual I/T ratio between current study and large cohort studies in literature.

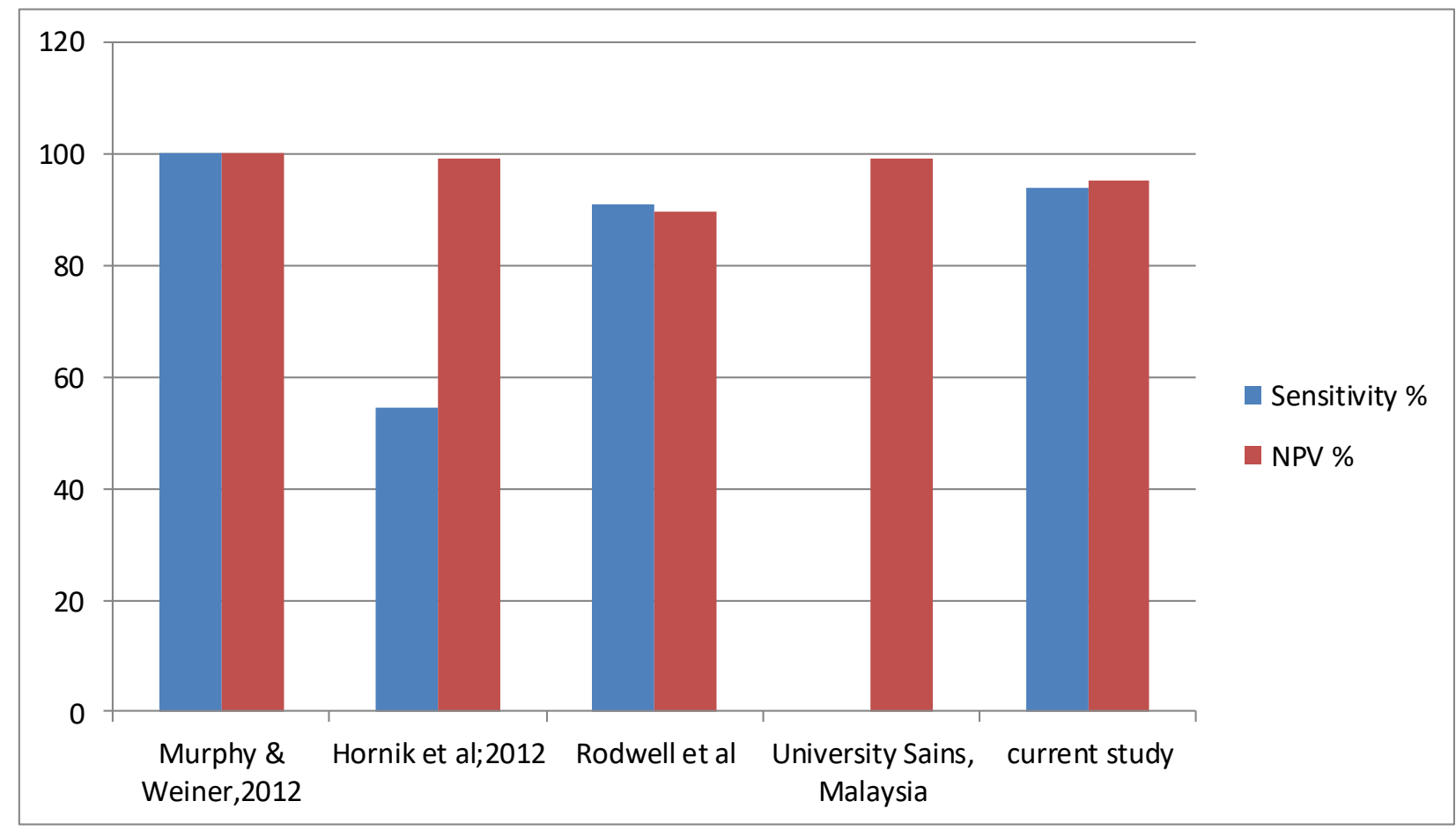

performed using the Coulter Act Diff 5 counter. Higher values of IGC $>0.3$ and IG\%>3 had a specificity more than $90 \%$ although the values were infrequent. Data for neonates separately was not available in this study.

However, sensitivity for IG\% $0.5 \%$ was unacceptably low with $33 \%$ in study "Performance of immature granulocyte count as a predictor of neonatal sepsis". ${ }^{8}$ Due to the inconsistency and greatly varied sensitivity results given above, these cut-offs cannot be recommended.

Furthermore, in the current study the NPV for above cut-off values were very poor with $25.00 \%$ for IGC 0.03 and $42.86 \%$ for IG\% $0.5 \%$ reflecting this as a poor predictor in 
excluding neonatal sepsis. The NPV was improved with higher cut-off values with $70.90 \%$ for IGC 0.3 and $70.59 \%$ for IG\% 3\%. Again the use of these cut-off values deemed unsuccessful with lower sensitivity of $40.00 \%$ and $33.33 \%$ respectively.

For automated IGC and IG\% none of the above cut-off values have shown validity to use in diagnosis of early neonatal sepsis in current study or in literature. This points out the importance of further large cohort studies formulating specific reference ranges for neonates (or paediatric age groups), with proper validation and quality control.

\section{CONCLUSION}

Immature to total neutrophil (I/T) ratio remains as a useful diagnostic tool in diagnosing and excluding neonatal sepsis with a very good sensitivity and NPV. However, further studies with improvement in method of detecting immature granulocytes and well defined reference intervals are required in automated IGC and IG\%. As the sample size is small in our study, a future study with a larger cohort is highly recommended to draw better conclusions. 


\section{DECLARATION}

\section{Ethics approval and consent to participate}

FBC analysis, blood culture and CRP consisted part of the investigations in a diagnostic work up of a baby with suspected sepsis. For other babies who were admitted to the neonatal unit for various reasons, FBC analysis was a routine test. Therefore, there were no additional procedures done on the patient to obtain samples. However, ethical clearance for the study was obtained from the Ethical Review Committee, Colombo South Teaching Hospital, Sri Lanka prior to starting the study.

Written informed consent was obtained from mother of each neonate before the commencement of the study. When gathering data, personal identifications was not used to ensure their privacy and confidentiality. Data was not accessible to any third party other than the research team. The research was carried out in accordance with the standard international guidelines on human studies.

\section{Consent for publication}

Not applicable as no personal identification details were used in the research paper.

\section{Availability of data and materials}

Can be produced to the journal editor on request.

\section{Competing interests}

All authors declare no conflict of interest.

\section{Funding}

No grants were obtained.

Analytical instruments private limited gave us financial support to complete this project. 


\section{Authors' contributions}

Author NJ wrote the proposal, managed the literature survey, carried out the data collection, performed data analysis and wrote the first draft of the manuscript. Author CK designed the study, corrected the proposal and literature survey, supervised and guided methodology and data analysis and corrected and modified the final manuscript up to this version. Authors SW and IW supervised the proposal writing and guided the data collection. All the authors read and approved the final version of the manuscript.

\section{Acknowledgements}

We would like to thank Dr. Rajiv Senthilnadajahal, Consultant Paediatricians and the staff in SCBU and Post-natal ward, Colombo South Teaching Hospital(CSTH), for the support given in data and sample collection, Ms.W.M.C.W.Manike, Mr.W.A.T.P Ranasinghe and other staff members at haematology laboratory, professorial unit, CSTH and the staff at Haematology and Microbiology laboratories at National Cancer Institute Maharagama(NCIM), for data collection and sample analysis.

We would also like to thank Dr. Sashikala Suresh, Consultant Haematologist and Dr. Samanmali Gunasekara, Consultant Microbiologist at NCIM for giving permission to analyze samples in the relevant automated machines.

We would also thank Analytical instruments (PVT) Ltd. for supporting with quality control material and reagents for the study.

\section{Authors' information (optional)}

Dr. Nalika Jayasekara

Senior Registrar in Haematology

Department of Pathology, Faculty of Medical Sciences, University of Sri Jayewardenepura, Sri Lanka 
Dr.Chandima Kulathilake,

Consultant Haematologist/Senior Lecturer

Department of Pathology, Faculty of Medical Sciences, University of Sri

Jayewardenepura, Sri Lanka

Saraji Wijesekara,

Consultant Paediatrician/ Senior Lecturer

Department of Paediatrics, Faculty of Medical Sciences, University of Sri

Jayewardenepura, Sri Lanka

Indira Wijesiriwardena

Consultant Haematologist/Senior Lecturer

Department of Pathology, Faculty of Medical Sciences, University of Sri

Jayewardenepura, Sri Lanka 


\section{TABLES}

Table 1: Distribution of the sample according to the diagnosis

\begin{tabular}{|l|l|l|}
\hline Category & Number & Percentage \\
\hline FBC done for other reasons & 6 & $11.76 \%$ \\
\hline Investigated for sepsis, but not treated with Antibiotics & 6 & $11.76 \%$ \\
\hline Investigated and treated with antibiotics, no proven sepsis & 20 & $39.22 \%$ \\
\hline Culture negative proven sepsis & 18 & $35.30 \%$ \\
\hline Culture positive sepsis & 1 & $1.96 \%$ \\
\hline Total & $\mathbf{5 1}$ & $\mathbf{1 0 0} \%$ \\
\hline
\end{tabular}

Table 2: Number of samples analyzed for manual I/T, automated IGC and IG\%

\begin{tabular}{|l|l|}
\hline Mode of analysis & Number \\
\hline Manual I/T ratio only & 11 \\
\hline $\begin{array}{l}\text { Both manual I/T ratio and automated IGC and } \\
\text { IG\% }\end{array}$ & 31 \\
\hline Automated IGC and IG\% only & 09 \\
\hline Total & $\mathbf{5 1}$ \\
\hline
\end{tabular}


Table 3: I/T ratio for manual method

\begin{tabular}{|l|l|l|l|}
\hline \multicolumn{1}{|c|}{ Final Diagnosis } & Sepsis & No sepsis & Total \\
\hline Positive $(\geq 0.2)$ & $15(\mathrm{TP})$ & $06(\mathrm{FP})$ & 21 \\
\hline Negative $(<0.2)$ & $01(\mathrm{FN})$ & $20(\mathrm{TN})$ & 21 \\
\hline Total & 16 & 26 & 42 \\
\hline
\end{tabular}

Sensitivity $=\mathrm{TP} /(\mathrm{TP}+\mathrm{FN}) \times 100 \%$

$$
\begin{aligned}
& =93.75 \% \\
\mathrm{NPV} \quad=\mathrm{TN} /(\mathrm{TN}+\mathrm{FN}) \times 100 \% & \\
= & 95.24 \%
\end{aligned}
$$


Table 4: Automated IGC for cut-off 0.03

\begin{tabular}{|l|l|l|l|}
\hline \multicolumn{1}{|c|}{ Final } & & \\
\hline IGC $(0.03)$ & & No sepsis & Total \\
\hline Positive $(>0.03)$ & $12(\mathrm{TP})$ & $24(\mathrm{FP})$ & 36 \\
\hline Negative $(\leq 0.03)$ & $03(\mathrm{FN})$ & $01(\mathrm{TN})$ & 04 \\
\hline Total & 15 & 25 & 40 \\
\hline
\end{tabular}

Sensitivity $\%=\mathrm{TP} /(\mathrm{TP}+\mathrm{FN}) \times 100 \%$

$$
=80.00 \%
$$

$\mathrm{NPV} \%=\mathrm{TN} /(\mathrm{TN}+\mathrm{FN}) \times 100 \%$

$$
=25.00 \%
$$


Table 5: Automated IGC for the cut-off of 0.3

\begin{tabular}{|l|l|l|l|}
\hline IGC $(0.3)$ & Sepsis & No sepsis & Total \\
\hline Positive $(>0.3)$ & $06(\mathrm{TP})$ & $03(\mathrm{FP})$ & 09 \\
\hline Negative $(\leq 0.3)$ & $09(\mathrm{FN})$ & $22(\mathrm{TN})$ & 31 \\
\hline Total & 15 & 25 & 40 \\
\hline
\end{tabular}

Sensitivity $\%=\mathrm{TP} /(\mathrm{TP}+\mathrm{FN}) \times 100 \%$

$$
\begin{aligned}
& =40.00 \% \\
\mathrm{NPV} \% & =\mathrm{TN} /(\mathrm{TN}+\mathrm{FN}) \times 100 \% \\
& =70.90 \%
\end{aligned}
$$

Table 6: Comparison of Sensitivity and NPV of automated IGC for the cut-off values of 0.03 and 0.3

\begin{tabular}{|l|l|l|}
\hline Cut off value for IGC & Sensitivity & NPV \\
\hline 0.03 & $80.00 \%$ & $25.00 \%$ \\
\hline 0.3 & $40.00 \%$ & $70.90 \%$ \\
\hline
\end{tabular}


Table 7: Automated IG\% cut-off value $0.5 \%$

\begin{tabular}{|l|l|l|l|}
\hline IG\% $(0.5 \%)$ & Final & No sepsis & Total \\
\hline Positive $(>0.5 \%)$ & $11(\mathrm{TP})$ & $22(\mathrm{FP})$ & 33 \\
\hline Negative $(\leq 0.5 \%)$ & $04(\mathrm{FN})$ & $03(\mathrm{TN})$ & 07 \\
\hline Total & 15 & 25 & 40 \\
\hline
\end{tabular}

Sensitivity $\%=\mathrm{TP} /(\mathrm{TP}+\mathrm{FN}) \times 100 \%$

$$
\begin{aligned}
& =73.33 \% \\
\mathrm{NPV} \% & =\mathrm{TN} /(\mathrm{TN}+\mathrm{FN}) \times 100 \% \\
& =42.86 \%
\end{aligned}
$$

Table 8: Automated IG\% for the cut-off $3 \%$

\begin{tabular}{|l|l|l|l|}
\hline IG\% (3\%) & & \\
\hline Positive $(>3 \%)$ & $05(\mathrm{TP})$ & $01(\mathrm{FP})$ & 06 \\
\hline Negative $(\leq 3 \%)$ & $10(\mathrm{FN})$ & $24(\mathrm{TN})$ & 34 \\
\hline Total & 15 & 25 & 40 \\
\hline
\end{tabular}


Sensitivity $\%=\mathrm{TP} /(\mathrm{TP}+\mathrm{FN}) \times 100 \%$

$$
=33.33 \%
$$

$\mathrm{NPV} \%=\mathrm{TN} /(\mathrm{TN}+\mathrm{FN}) \times 100 \%$

$$
=70.59 \%
$$

Table 9: Comparison of sensitivity and NPV for IG\% of $0.5 \%$ and $3 \%$

\begin{tabular}{|l|l|l|}
\hline Cut off value for IG\% & Sensitivity & NPV \\
\hline 0.5 & $73.33 \%$ & $42.86 \%$ \\
\hline 3 & $33.33 \%$ & $70.59 \%$ \\
\hline
\end{tabular}




\section{REFERENCES}

1. Hornik CP, Benjamin DK, Becker KC, Benjamin DK Jr, Li J, Clark RH, CohenWolkowiez M, Smith PB. Use of the complete blood cell count in early-onset neonatal sepsis. Pediatr Infect Dis J,2012 Aug;31(8);799-802.

2. Makkar M, Gupta C, Pathak R, Garg S, Mahajan NC. Performance Evaluation of Hematologic Scoring System in Early Diagnosis of Neonatal Sepsis. J Clin Neonatol. 2013 Jan;2(1):25-9.

3. Ghosh S, Mittal M, Jaganathan G. Early diagnosis of neonatal sepsis using a hematological scoring system. Indian J Med Sci 2001;55:495-500.[ PubMed ]

4. Narasimha A, Harendra Kumar ML. Significance of Hematological Scoring System (HSS) in early diagnosis of neonatal sepsis. Indian J Hematol Blood Transfus 2011;27:14-7.

5. Murphy K, Weiner J. Use of Leukocyte Counts in Evaluation of Early-onset Neonatal

Sepsis. Pediatr Infect Dis J. 2012 Jan;31(1):16-9.

6. Rodwell RL, Leslie AL, Tudehope DI. Early diagnosis of neonatal sepsis using a hematologic scoring system. J Pediatr. 1988;112:761-767.

7. Dr. Rosline Hassna. Evaluation of IG and IT ratio in cases with presumed neonatal sepsis. Hospital Universiti Sains MALAYSIA, Kelantan. (December 2012).

8. Kelly G., Nigro, MD, MaryAnn O'Riordan, MS, Eleanor J. Molloy, MD, PhD, Michele C. Walsh, MD, MS, and Linda M. Sandhaus, MD, MS. Performance of an Automated Immature Granulocyte Count as a Predictor of Neonatal Sepsis. American Journal of Clinical Pathology 2005.

9. Belamurugan Senthilnayagam, Treshul Kumar, Jayapriya Sukumaran, Jeya M., and Ramesh Rao K. Automated Measurement of Immature Granulocytes: Performance characteristics and Utility in Routine Clinical Practice. Chettinad 
Deemed University, Padur, India. (Pathology Research International, volume 2012).

10. Walliullah SM, Islam MN, Siddika M, Hossain MA, Chowdhury AK. Role of micro-ESR and

I/T ratio in the early diagnosis of neonatal sepsis. Mymensingh Med J, 2009 Jan;18(1);56-61.[ PubMed ].

11.Walliullah SM, Islam MN, Siddika M, Hossain MA, Jahan I, Chowdhury AK.

Evaluation of simple hematological screen for early diagnosis of neonatal sepsis.

Mymensingh Med J, 2010 Jan;19(1);41-7.[ PubMed ]

12.Selimović A, Skokić F, Selimović Z, Bazartzanović M. [The predictive values of total white bood count and differential count in the diagnosis of early-onset neonatal sepsis].Med Arh 2008;62(4);205-10.

13.Ahmed Z, Ghafoor T, Waqar T, Ali S, Aziz s, Mahmud S. Diagnostic value of Creactive protein and haematological parameters in neonatal sepsis.J Coll Physicians Surg Pak, 2005 Mar;15(3);152-6.[ PubMed ] 
Figures

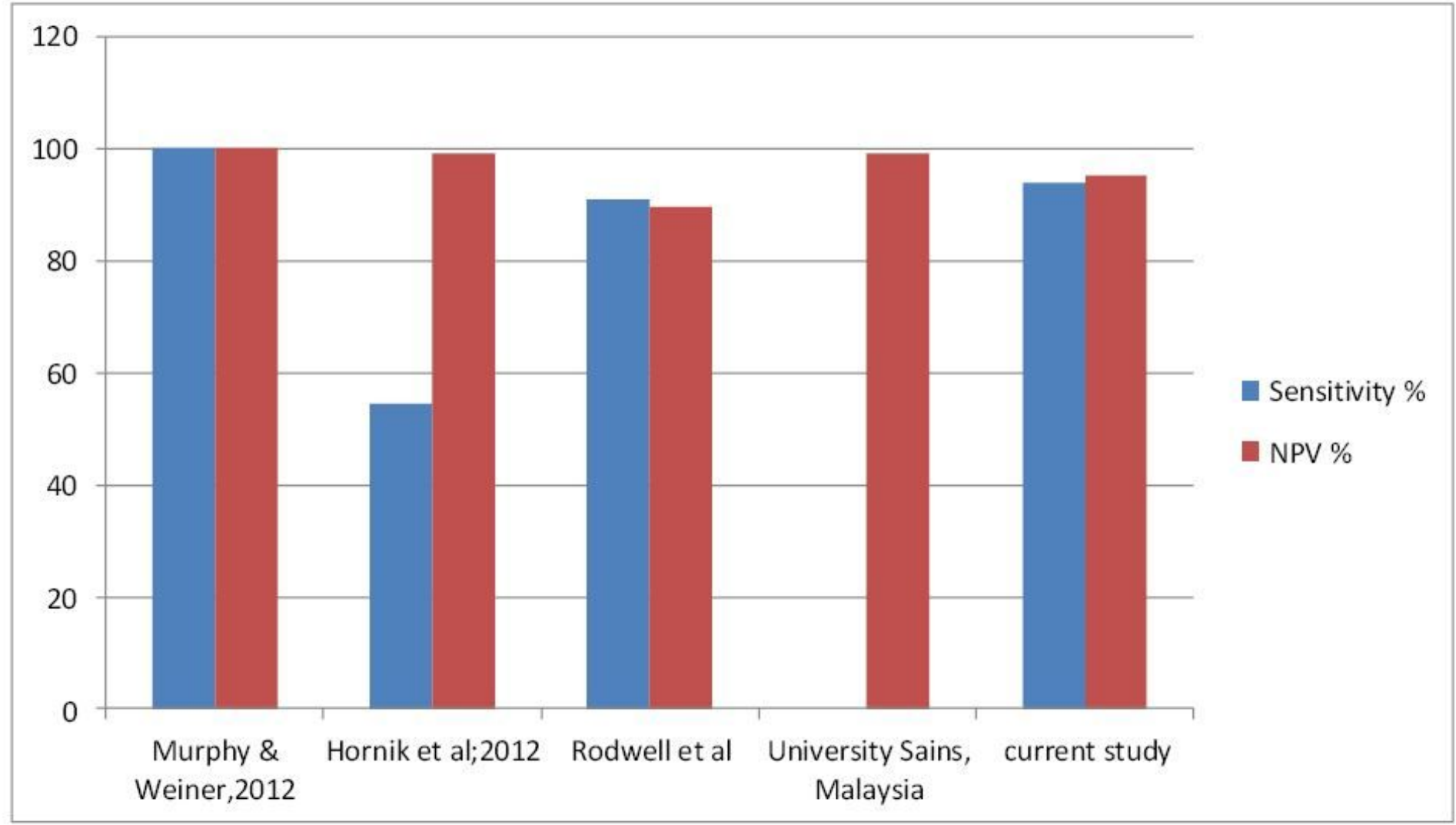

Figure 1

Comparison of Sensitivity and NPV of manual I/T ratio between current study and large cohort studies in literature. 